\title{
The effects of gene-environment interaction on the expression of longevity
}

\author{
Michael J. Clare and \\ Leo S. Luckinbill
}

Department of Biological Sciences, Wayne State

University, Detroit, Michigan, 48202.

Several previous attempts at selecting for increased life span with Drosophila have failed to obtain a response to selection, and postulate that life span is controlled by non-genetic maternal effects instead of genes. In other experiments, however, populations have responded. This study uses a set of true-breeding long- and short-lived stocks developed by in vitro selection to examine the effect on life span of developmental environment and outcrossing.

The expression of genes for life span is shown here to vary as a result of a gene-environment interaction and is strongly affected by the environment during development. The longevity of $F_{1}$ crosses between long- and short-lived parental lines is additive when the density of larvae is high and unfixed, showing that life span is controlled by genes and not nongenetic maternal effects. But, when numbers of larvae are held low and constant, as in studies where selection fails, life span of crosses and parental lines is greatly restricted. The failure of some previous attempts at selection, therefore, appears to have resulted from the introduction, through experimental design, of strong artifactual environmental effects that limit the phenotypic expression of genes for life span and the effectiveness of selection.

Comparison of fecundity in parental and $F_{1}$ lines shows that selection for increased life span antagonistically reduces early-life fecundity. Short-lived, parental lines, reproduced at an early age in life, lay $22-24$ per cent more eggs in the same period than do long-lived lines selected for reproduction at a late age.

\section{INTRODUCTION}

More than two decades ago the first predictive, genetically based interpretations of the phenomenon of senescence were proposed in which the aging process was not itself considered to be adaptive. Medawar (1952) first formally introduced the notion that specialised age-of-onset modifier genes suppress the action of deleterious genes until late in the life of the organism when the force of natural selection is diminished and the expression of harmful genes carries little penalty. Their eventual release from inhibition determines the onset of senescence. Natural selection, therefore, is seen to act on life span by altering the action of modifier genes.

Williams (1957), on the other hand, added the explicit consideration of pleiotropy to the previous model with the proposal that genes controlling senescence are both beneficial early in life and detrimental late in life. In particular, early- and late-fitness should be associated such that an enhanced early-fitness would be accompanied by a more rapid onset of senescence and shorter life. Selection favouring an increased longevity or latefecundity would occur at the expense of fecundity early in life.

Though these theories differ in several major respects, relating to the mechanism of genetic control each proposes (Charlesworth, 1980), they both make the fundamental assumption that longevity is under genetic control and modifiable by natural selection. Selection favouring reproduction by individuals at an advanced age should increase life span, while reproduction by the young should hold life span low or reduce it.

Recent experiments, however, contest even these reasonable assumptions. In a comprehensive series of studies with Drosophila melanogaster, Lints and Hoste $(1974 ; 1977)$ and Lints et al., (1979) applied selection for increased life span under different regimes of age-specific reproduction with the expectation that longevity would increase in lines reproduced at a late age in life. 
But their results were so unusual that no genetically based theory of aging could explain them. Longevity fluctuated widely and underwent several spontaneous reversals during selection. Because of the similarity between their results and those of Lansing (1947; 1954), Lints and Hoste (1974) concluded that life span is not controlled by genes, but by nongenetic factors of maternal origin.

Other selection experiments by Rose (1984) and Rose and Charlesworth $(1980 ; 1981)$ and Luckinbill et al. (1984), however, are consistent with evolutionary theories based on genetic control, and present evidence in support of Williams' (1957) notion of pleiotropic gene action.

In this study, we use the long-lived lines developed by Luckinbill and Clare (1985) in crosses with short-lived lines from the same origin to obtain an $F_{1}$ generation. By comparing fecundity and longevity in parental and $F_{1}$ populations, two predictions of theories of aging are tested, as well as a third concerning the influence of artifactual effects in these experiments.

First, we explicitly test for genetic versus longenetic control of life span by comparing longevity in the $F_{1}$ generation with that of the parents. If life span is under the nongenetic control of the maternal parent, as Lints and Hoste (1974) propose, then longevity in the $F_{1}$ will correspond to that of the respective maternal parents and will not reflect its genetic composition by any conventional expression of dominance or additivity. And second, we further test the conclusion of Luckinbill and Clare (1985) that controlling population density during development determines the life span expressed in adults. And finally, we attempt to distinguish between existing genetic theories of aging, particularly by testing Williams' (1957) prediction that antagonistic pleiotropy should occur between early- and late-life components of fitness. Here the early-life fecundity of long- and shortlived parental lines are compared.

\section{METHODS}

The long-lived parental strain used here was taken from the lines of the uncontrolled-density treatment of Luckinbill and Clare (1985) at generations 16 and 22. Populations of that study were collected locally in Michigan and following the method of Lints and Hoste (1974), were used to create a four-way hybrid stock. This basic stock was maintained for eight generations before selection was begun. Selection consisted of reproduction at either an early age (days 4-6 after eclosion) or a late age in life. The age of late-reproduction was initially set at from days 22 to 26 . But as selection progressed this was varied, such that by the $21 \mathrm{st}$ generation, late reproduction was occurring at over 70 days after eclosion. At the termination of that experiment, the adult life span of late-reproducing lines had advanced by as much as 56 per cent over that of lines reproduced at an early age.

Because of the difference in generation times imposed by selection for reproduction at early versus late ages, lines under early-selection of that experiment had completed 29 generations of selection long before late-reproducing lines had even reached generation 21 . Therefore, in order to adapt those long-lived lines for this study and perform crosses with short-lived lines subjected to equivalent selection, two separate sets of earlyreproducing lines were started from the original hybrid stock and timed to synchronise with approximately the 16th and 21 st generations of selection for late-reproducing lines in that experiment. Thus, crosses here were between early- and late-reproducing lines selected for the same number of generations.

Before crossing the long and short-lived lines, the two replicates of each respective selection treatment were used to generate a single line serving as parent population of that treatment. To create the short-lived parental line, 50 pairs of males and females of the two replicate early-selection lines were reciprocally crossed to each other. A single long-lived parental line was created in an identical fashion by crossing the two long-lived (late-reproducing) selection lines. The parental lines of this study are, therefore, themselves the outcrossed product of replicate long- or short-lived selection lines.

To produce the $F_{1}$ generation, populations of 50 females from the long- and short-lived parental lines were reciprocally crossed to males $8-10$ days after eclosion. This procedure was followed twice, using late-reproducing lines from the 16th and again at the 22 nd generation of selection of Luckinbill and Clare (1985). Because no significant differences were observed between reciprocal populations crossed in this way, the final $F_{1}$ crosses of this study were produced by a single composite population consisting of 25 reciprocally crossed females from each parental line, combined for egg laying, into a single population of 50 . Both parental and $F_{1}$ lines were maintained on media and culture conditions described by Luckinbill and Clare (1985). Longevity and fecundity were measured for populations of 30 paired females, also as described there. Larvae were raised without 
density-control, except where specified. Development was constant at 11-12 days for all lines except for populations raised under controlled-density conditions, where development was 9-10 days. Estimates of longevity are for total longevity, and include development.

To determine the extent to which the larval environment affects the expression of life span in adults, $F_{1}$ individuals collected from the second measurement of parental lines and crosses were also raised at a constant larval density of 10 per standard shell vial, as in Lints and Hoste (1974) and Lints et al. (1979). Except for their density at development, these lines were identical in both composition and treatment to the other $F_{1}$ line from that cross. Parental lines were not raised under controlled-density conditions in the second cross. In the final comparison, however, both $F_{1}$ and parental lines were raised under controlled and uncontrolled treatments to show the simultaneous effect on all populations of larval-density control. Populations were raised at controlled densities of 10,30 and 50 larvae per vial. Parental lines for these crosses were drawn respectively from the 20th and 23rd generation of selection for early-and late-reproduction.

\section{RESULTS}

The survivorship and average total life span of long- and short-lived parental lines, and the first replicate crosses between them are shown in fig. 1. Viability was not estimated in this study and percentage survivorship is measured from eclosion only. The long-lived parental line of this cross was from generation 16 of Luckinbill and Clare (1985). Reciprocal $F_{1}$ populations are clearly intermediate in longevity between long- and short-lived parental lines, and near the midparent value for such a cross.

The analysis of life span in this cross is shown in table $1 \mathrm{~A}$ and B. A Student-Newman-Kuhls test indicates that mean life span in $F_{1}$ populations are neither significantly different from one another

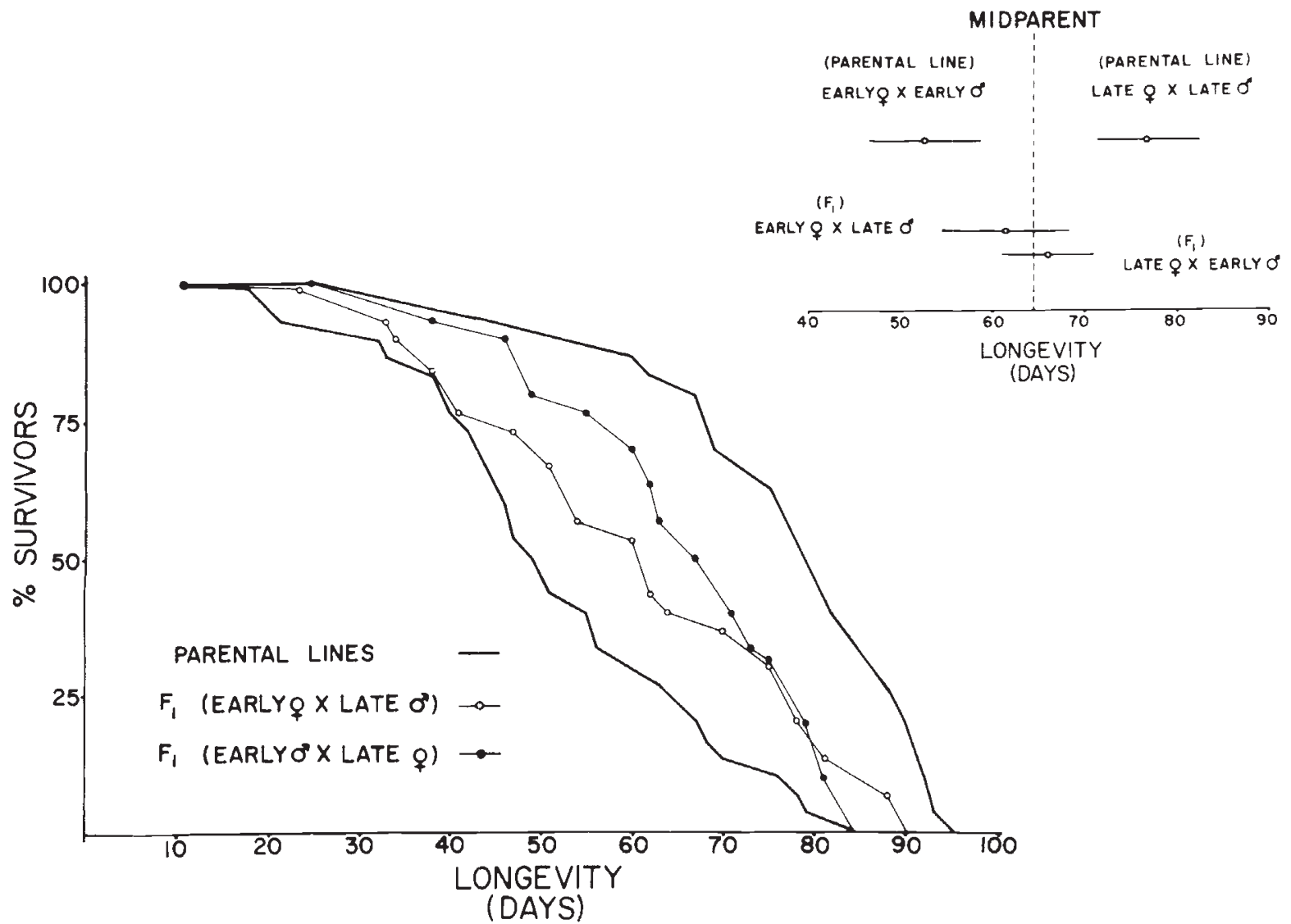

Figure 1 Survivorship from eclosion of long- and short-lived parental lines and reciprocal $F_{1}$ crosses are shown at lower left. The insert (upper right) shows the mean (circles) and 95 per cent confidence intervals for total longevity of the populations. 
Table 1 The longevities of replicate parental and $F_{1}$ lines are shown in (A). Longevities of $F_{1}$ lines when raised with larval density controlled are also shown. The grouping and analysis by Student-Newman-Kuhls Test is indicated in (B)

(A)

LONGEVITY

REPLICATE

12

Parental Lines

$\begin{array}{llcr}\text { EARLY-REPRODUCED } & \text { (E) } & 52.2 & 54.6 \\ \text { LATE-REPRODUCED } & \text { (L) } & 77.0 & 78.0 \\ & & & \\ & & \text { F Lines }\end{array}$

EARLY-REPRODUCED $\$ \%$

LATE-REPRODUCED $\delta^{\circ} 0^{\circ}$

DENSITY CONTROLLED (dexdI) - $\quad 56.5$

LATE-REPRODUCED $\$ \%$

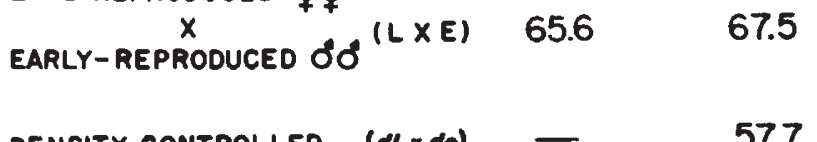

DENSITY CONTROLLED $(d 1 \times d d) \quad-\quad 57.7$
(B)

GAOUPINE OY

StUdent-NemMan-Kuhls test
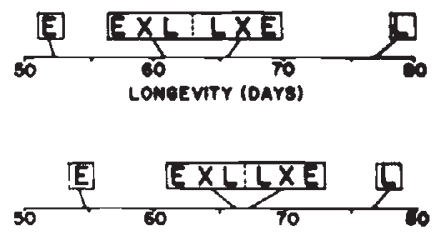

DENSITY CONTROLLED DURING DEVELOPMENT
66.2

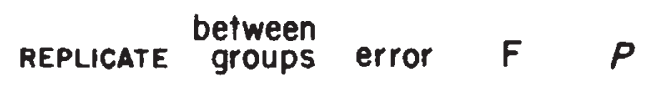

1

$3184.5 \quad 262.8 \quad 12.2$

2

$2368.9 \quad 185.9 \quad 12.8$ nor from the midparent value for parental lines. They are, however, significantly different from both long- and short-lived parental lines.

The additive expression of longevity is also clearly evident in the second and subsequent replicate cross of long and short-lived lines in fig. 2 . Parentals here were taken from generation 22 of Luckinbill and Clare (1985). Longevity of parental lines is very similar to that of the first replicate and the mean life span for the $F_{1}$ is also very close to the midparent value for this trait. But, as the insert for fig. 2 also indicates, when $F_{1}$ populations are raised under controlled-density conditions as larvae, life span is substantially decreased to the level of the short-lived parental line.

The analysis of longevity in parental lines and density-treatments of this cross is shown in table 1B. Populations raised with developmental density uncontrolled show the same relationships as in the first replicate, with $F_{1}$ populations significantly different from either parental line, but not from the midparent value nor one another. The additivity shown by genes for longevity in the first cross is therefore, repeatable.

As shown by the $F_{1}$ populations in figs. 2 and 3 , the control of larval density alters adult life span in both parental and $F_{1}$ lines. All populations raised at a low controlled-density have a substantially lower longevity than when uncontrolled, and are very close to the short-lived parental line. As fig. 3 shows, mean longevity is reduced in the long-lived parental line from 78.53 days to 63.56 days, which is less than the midparent value for the uncontrolled treatment. $F_{1}$ populations at densities of 10 and 30 per vial are significantly depressed below the midparent value $(0.05>P>$ $0.025, t=-1.755$, one-tailed test and $0.01>P>$ $0.005, t=-3.092$ respectively). The $F_{1}$ population raised at 50/vial regains additivity, however, lying again on the midparent value. Thus, when the developmental density is held low, as in the experiments of Lints and Hoste (1974) and Lints et al. 


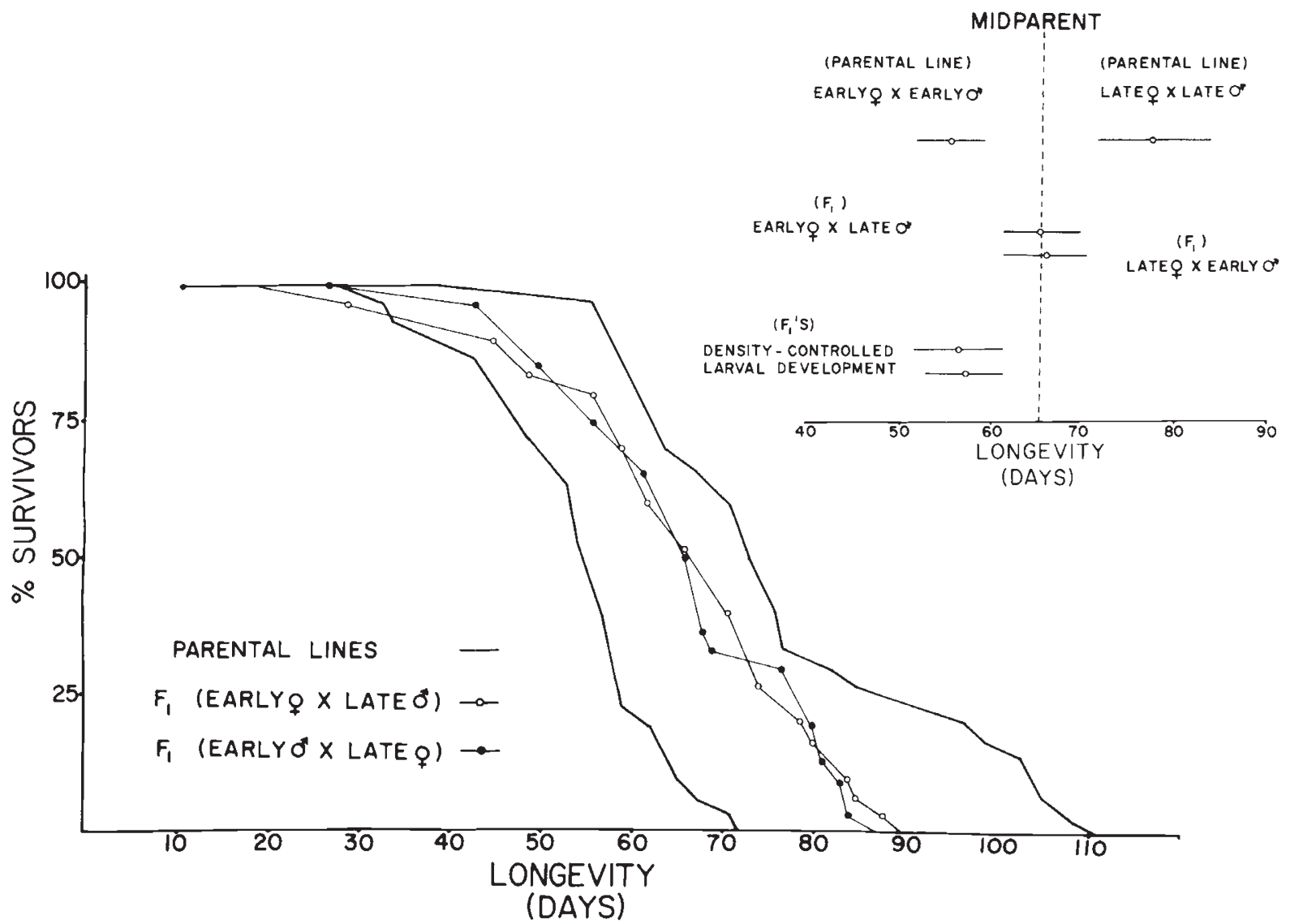

Figure 2 Survivorship from eclosion of parental lines and $F_{1}$ crosses are at left. The insert (upper right) shows the mean and 95 per cent confidence intervals of total longevity in the populations. The mean and confidence interval are also shown for $F_{1}$ crosses raised with their larval-density controlled. Survivorship curves are not shown for these populations.

(1979), adult longevity comes under some strong form of limitation. Longevity in all long-lived lines is reduced to nearly that of short-lived control lines. Increasing the density of $F_{1}$ populations to a high level can restore additivity even though development occurs in vials and density is controlled.

Table 2 compares the early-life fecundity of long- and short-lived parental populations and $F_{1}$ crosses. Fecundity was measured on days 3-5 of adult life to conform with the age at which selection for early-reproduction took place, as in Luckinbill et al. (1984). Early-fecundity varies in successive replicates, but is consistently less in long-lived parental lines than in short-lived lines. Short-lived parental lines lay 22 or 24 per cent more eggs in the same period than do lines selected for latereproduction and long life. For $F_{1}$ populations, however, the pattern is less clear. $F_{1}$ crosses show neither additivity, nor is their pattern repeatable across replicates. One population of the reciprocal cross in each case proves to be overdominant, while the other is intermediate between parental lines.

\section{CONCLUSIONS}

This study shows that:

(1) Genes do control life span and are expressed additively in $F_{1}$ crosses between extremes of selected lines, when the density of developing larvae is uncontrolled.

(2) When numbers of developing larvae are held low, populations show only a short adult life regardless of their genetic capability.

(3) 16-22 generations of selection for late-reproduction extends life span and antagonistically reduces early-fecundity. Short-lived lines under selection for early-reproduction lay 2224 per cent more eggs in the same period than long-lived lines. 


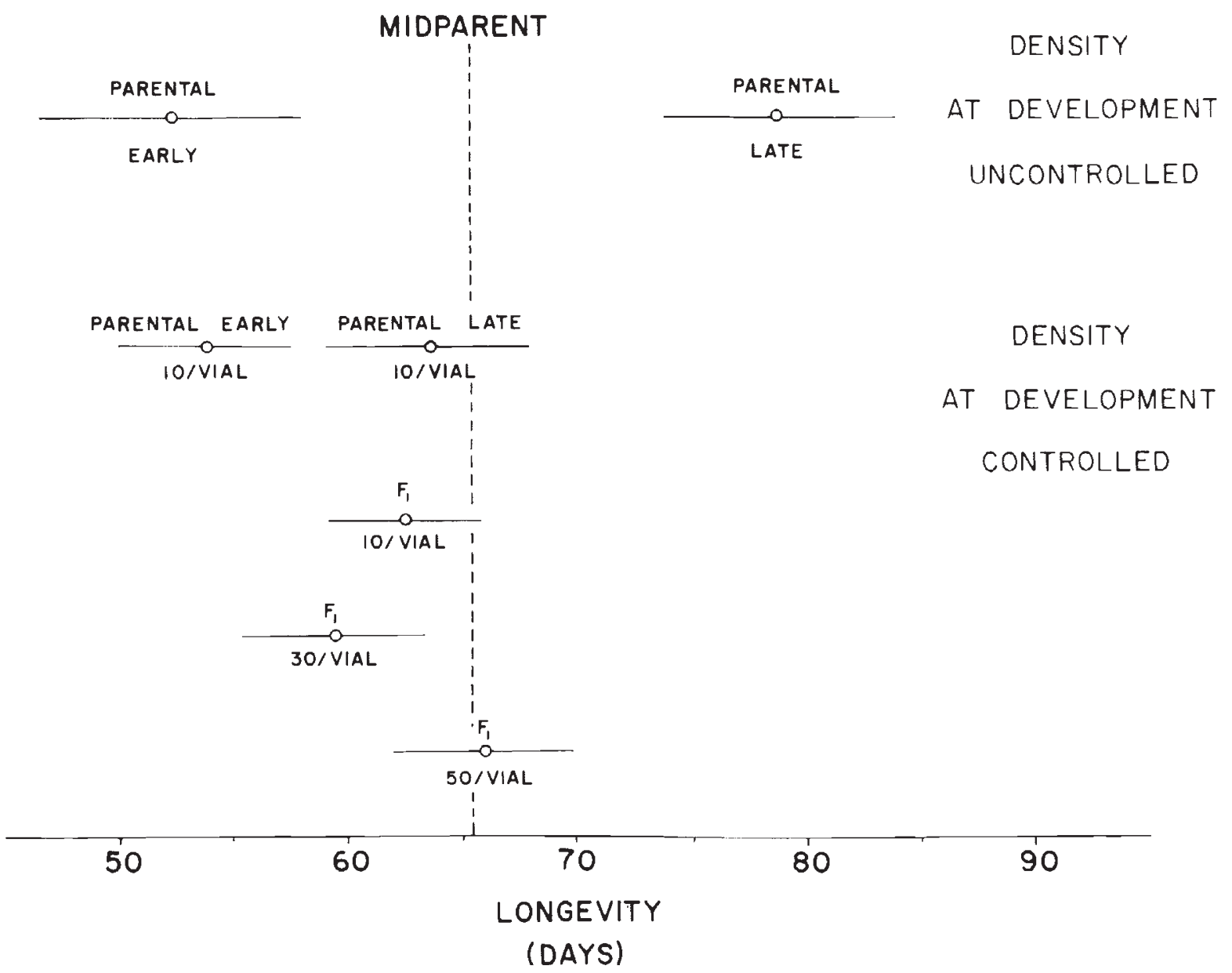

Figure 3 Means and 95 per cent confidence intervals of longevity are shown for populations raised under both experimental treatments. Longevity is compared in long- and short-lived stocks raised at an uncontrolled developmental-density and for populations reared at densities of 10,30 and 50 larvae/vial.

Table 2 Means and variances of early-fecundity (days 3-5 of adult life) are shown for replicate parental lines and $F_{1}$ crosses. Early-fecundity in parental lines is analyzed by $t$-Test

\begin{tabular}{|c|c|c|c|c|c|c|c|}
\hline \multirow[b]{3}{*}{ Replicate } & \multicolumn{4}{|c|}{$\begin{array}{c}\text { Early fecundity days } 3-5 \text { of adult life } \\
\text { Parental lines }\end{array}$} & \multirow[b]{3}{*}{$t$} & \multirow[b]{3}{*}{$N$} & \multirow[b]{3}{*}{ Significance } \\
\hline & \multicolumn{2}{|c|}{$\begin{array}{l}\text { Early- } \\
\text { reproduced }\end{array}$} & \multicolumn{2}{|c|}{$\begin{array}{l}\text { Late- } \\
\text { reproduced }\end{array}$} & & & \\
\hline & mean & $S$ & mean & $S$ & & & \\
\hline \multirow{2}{*}{$\begin{array}{l}1 \\
2\end{array}$} & $87 \cdot 6$ & $30 \cdot 8$ & $71 \cdot 7$ & 36.4 & 1.82 & 58 & $p<0.05$ \\
\hline & $\begin{array}{l}\text { Early- } \\
\text { Late-r }\end{array}$ & $\begin{array}{l}\text { uced } q 9 \\
\operatorname{ced} \delta \delta\end{array}$ & $\begin{array}{r}\quad F \\
\text { Late-r } \\
\text { Early- }\end{array}$ & $\begin{array}{l}\text { ced } \$ 9 \\
\text { uced } \delta 8\end{array}$ & & & \\
\hline Replicate & mean & $S$ & mean & $S$ & & & \\
\hline
\end{tabular}


Lints and Host (1974) and Lints et al. (1979) present careful, well designed experiments selecting for long and short life in D. melanogaster that do not conform to even the most basic assumption of theory. Such experiments might be overlooked with easy $A D H O C$ explanations, except that they are repeatable. The control of larval density in the study by Luckinbill and Clare (1985) shows strikingly similar deviations, with the same overall nonresponse to selection that several previous studies have found (Lints and Hoste, 1974; Lints et al., 1979; Lints and Gruwez, 1972; and Flanagan, $1980)$.

Lacking any evidence to the contrary, Lints and his co-workers conclude that longevity is not controlled by genes but by nongenetic maternal effects, as Lansing found $(1947 ; 1954)$. Our experiments, however, show that maternal effects are not the determinants of longevity, and confirm an alternative explanation, as yet unconsidered. Namely, we suggest the implacability of life span under their conditions to be artifactual, and caused by a peculiarity of the experimental conditions themselves; selection for increased longevity is resisted by the short-lived phenotype expressed under those conditions. We suggest that genes controlling longevity are conventional in every sense, even showing near ideal additivity in the uncontrolled-density treatment, and like other genes, their expression also includes an environmental component, significantly touched upon here by developmental conditions.

It is the expression of genes, and not the genes themselves which differ in the density-treatments of this study. Two mechanisms could accomplish this. First, development at low density may limit the permissible adult life span in some unknown physiological manner. In this case, different selection treatments would be ineffective and long- and short-lived stocks would be indistinguishable if placed there. In keeping with this, both the $F_{1}$ and long-lived lines of this study are found to be severely reduced by controlling developmental density. Yet, it is difficult to conceive what such a profound but subtle physiological effect might be, especially since environments of adult populations are identical, under selection or measurement, and developmental environments differ only by the number of larvae.

A second possibility, with similar predictions, is that the repression of long adult life by developmental density is genetic in origin. That is, in what appears to be nearly optimal conditions of controlled-density, variations in life span are suppressed in favour of the short-lived phenotype.
That a variable gene expression under one set of conditions would give way to a single less flexible phenotype under another, is not surprising in itself. Lerner (1954) and Waddington (1957; 1961), described at length the ideas of developmental buffering and threshold of character expression. Our results might constitute such a case. Genes sensitive to population density during development could have a threshold, expressing dominance at first, and finally additivity at higher density as development becomes more stressful. Several studies have shown that variation in gene expression occurs as a function of such geneenvironment interaction (Parsons, 1977; 1978; Derr, 1980; Murphy et al., 1983). Thus, ample theoretical and experimental support for this idea exists already, apart from the case we offer.

This hypothesis predicts that selection in experiments would eventually accomplish the derepression of the desired genes. Thus, it may be that the populations of Lints and his co-workers and Luckinbill and Clare (1985) would have eventually responded to selection. Indeed, the pattern of longevities in the final generations of those studies is not inconsistent with that possibility.

One of the main predictions of the pleiotropy theory of aging advanced by Williams (1957), is that selection improving late-fitness should reciprocally affect early-fitness. If the onset of senescence is controlled by pleiotropic elements linking early- with late-fitness, then selection for late-reproduction should lengthen life, but at the expense of "early vigour", as measured by fecundity. Rose (1984) and Rose and Charlesworth $(1980,1981)$ find a clear association between these features. Luckinbill et al. (1984) also found a significant but weaker trade-off of this sort. Earlyfecundity varies here between replicates, yet within a replicate, early-reproducing lines lay a consistent 22-24 per cent more eggs in early life than latereproducing lines. Thus, life span has been improved in these strains at considerable cost to early-fecundity.

Acknowledgements We thank Michael R. Rose for his comments on a previous draft of this work. This research was supported by grant AGO1812 from the National Institutes of Health (U.S.A.) to L.S.L. and Robert Arking.

\section{REFERENCES}

Charlesworth, B. 1980. Evolution in Age-structured Populations. Cambridge University Press, Cambridge. 
DERR, J. A. 1980. The nature of variation in life history characters of Dysdercus bimaculatus (Heteroptera: Pyrrhocoridae), a colonizing species. Evolution, 34, 548-557.

FLANAGAN, J. R. 1980. Detecting early-life components in the determination of the age of death. Mech. Aging Dev., 13, 41-62.

LANSING, A. 1. 1947. A transmissible, cumulative and reversible factor in aging. J. Gerontol., 2, 228-239.

LANSING, A. I. 1954. A nongenetic factor in the longevity of rotifers. Ann. N.Y. Acad. Sci., 57, 455-464.

Lerner, I. M. 1954. Genetic Homeostasis. Wiley, New York.

LINTS, F. A. ANI) GRUWEZ, G. 1972. What determines the duration of development in Drosophila melanogaster. Mech. Aging Dev., 1, 285-297.

LINTS, F. A. AND HOSTE, C. 1974. The Lansing Effect revisited. I. Life span. Exper. Gerontol, 9, 51-69.

LINTS, F. A. ANI HOSTE, C. 1977. The Lansing Effect revisited. II. Cumulative and spontaneously reversible effects on fecundity in Drosophila melanogaster. Evolution, 38(6), 996-1003.

LINTS, F. A., STOLL. J. A., GRUWEZ, G. AND LINTS, C. V. 1979. An attempt to select for increased longevity in Drosophila melanogaster. Gerontol, 5, 192-204.

LUCKINBILL, L. S., ARKING, R., CLARE, M. J., CIROCCO.W. AND BUCK, S. A. 1984. Selection for delayed senescence in Drosophila melanogaster. Evolution, 3(5), 9961003.
LUCKINBILL, L. S. AND CLARE, M. J. Selection for life span in Drosophila melanogaster. 1985. Heredity, 55, 9-18.

Medawar, P. B. 1952. An Unsolved Problem in Biology. Lewis, London.

MURPHY, P. A., GIESEL, J. T. AND MANLOVE, M. N. 1983. Temperature effects on life history variation in Drosophila simulans. Evolution, 37, 1181-1191.

PARSONS, P. A. 1978. The genetics of aging in optimal and stressful environments. Exp. Gerontol., 13, 357-363.

PARSONS, P. A. 1977. Genotype-temperature interactions for longevity in Drosophila simulans. Exp. Gerontol., 12, 241244.

ROSE, M. R. 1984. Laboratory evolution of postponed senescence in Drosophila melanogaster. Evolution, 38(5), 10041009.

ROSE, M. R. AND CHARL.FSWORTH, B. 1980. A test of evolutionary theories of senescence. Nature, 287, 141-142.

ROSE, M. R. AND CHARLESWORTH, B. 1981. Genetics of life history in Drosophila melanogaster. II. Exploratory selection experiments. Genetics, 97, 187-196.

Waddington, C. H. 1957. The strategy of the Genes. Allen and Unwin, London.

Waidington, C. H. 1961. Genetic Assimilation. Adv. Gen., $10,257-293$.

WILLIAMS, G. C. 1957. Pleiotropy, natural selection and the evolution of senescence. Evolution, 11, 398-411. 